Marquette University

e-Publications@Marquette

Biomedical Engineering Faculty Research and

Publications

Biomedical Engineering, Department of

2-12-2000

\title{
Micro-CT Image-Derived Metrics Quantify Arterial Wall \\ Distensibility Reduction in a Rat Model of Pulmonary Hypertension
}

\author{
Roger H. Johnson \\ Medical College of Wisconsin \\ Kelly L. Karau \\ College of Wisconsin \\ Robert C. Molthen \\ Marquette University, robert.molthen@marquette.edu \\ Steven Thomas Haworth \\ Medical College of Wisconsin \\ Christopher A. Dawson \\ Medical College of Wisconsin
}

Follow this and additional works at: https://epublications.marquette.edu/bioengin_fac

Part of the Biomedical Engineering and Bioengineering Commons

\section{Recommended Citation}

Johnson, Roger H.; Karau, Kelly L.; Molthen, Robert C.; Haworth, Steven Thomas; and Dawson, Christopher A., "Micro-CT Image-Derived Metrics Quantify Arterial Wall Distensibility Reduction in a Rat Model of Pulmonary Hypertension" (2000). Biomedical Engineering Faculty Research and Publications. 110.

https://epublications.marquette.edu/bioengin_fac/110 


\title{
Micro-CT image-derived metrics quantify arterial wall distensibility reduction in a rat model of pulmonary hypertension
}

\author{
${ }^{1,2,3,5}$ Roger H. Johnson, ${ }^{1}$ Kelly L. Karau, ${ }^{1,4,5}$ Robert C. Molthen, ${ }^{4,5}$ Steven T. Haworth, \\ and ${ }^{1,4,5}$ Christopher A. Dawson \\ ${ }^{1}$ Department of Biomedical Engineering, Marquette University \\ Departments of ${ }^{2}$ Radiology, ${ }^{3}$ Biophysics and ${ }^{4}$ Physiology, Medical College of Wisconsin \\ ${ }^{5}$ Research Service, Zablocki VA Medical Center \\ Milwaukee, Wisconsin, USA
}

\begin{abstract}
We developed methods to quantify arterial structural and mechanical properties in excised rat lungs and applied them to investigate the distensibility decrease accompanying chronic hypoxia-induced pulmonary hypertension. Lungs of control and hypertensive (three weeks $11 \% \mathrm{O}_{2}$ ) animals were excised and a contrast agent introduced before micro-CT imaging with a special purpose scanner. For each lung, four $3 \mathrm{D}$ image data sets were obtained, each at a different intra-arterial contrast agent pressure. Vessel segment diameters and lengths were measured at all levels in the arterial tree hierarchy, and these data used to generate features sensitive to distensibility changes. Results indicate that measurements obtained from 3D micro-CT images can be used to quantify vessel biomechanical properties in this rat model of pulmonary hypertension and that distensibility is reduced by exposure to chronic hypoxia. Mechanical properties can be assessed in a localized fashion and quantified in a spatially-resolved way or as a single parameter describing the tree as a whole. Micro-CT is a nondestructive way to rapidly assess structural and mechanical properties of arteries in small animal organs maintained in a physiological state. Quantitative features measured by this method may provide valuable insights into the mechanisms causing the elevated pressures in pulmonary hypertension of differing etiologies and should become increasingly valuable tools in the study of complex phenotypes in small-animal models of important diseases such as hypertension.
\end{abstract}

Keywords: arterial distensibility, arterial tree morphometry, vascular mechanics, biomedical x-ray imaging, micro-CT, microtomography, conebeam CT, pulmonary physiology

\section{INTRODUCTION}

There has been a long-standing interest in imaging-based methods to assess the "stiffness" of pulmonary arterial walls, whether this property was referred to as compliance ${ }^{1,2}$, elasticity", pulsatility", extensibility", incremental Young's modulus ${ }^{6}$ or distensibility ${ }^{7,8}$. Structural changes secondary to chronic hypoxia occur in the cardiac and pulmonary vessels of rats, causing profound deviations from the normal hemodynamic situation. Resistance in the peripheral arterial tree is significantly increased due to vessel wall remodeling' and/or small vessel obliteration ${ }^{\text {to }}$. Far higher pressures are required to maintain adequate flow, resulting in severe right heart hypertrophy and eventual death. Our goal is to gain insight into the causes and mechanisms involved in pulmonary hypertension of different etiologies by studying several small animal models of the disease ${ }^{11}$. We therefore require relatively high throughput methods to make quantitative measurements on small vessels in intact organs maintained in a near-physiological state.

In the past, experiments to measure vessel distensibilities have been conducted on individual, excised arterial segments ${ }^{8,12}$ or small portions of the arterial network ${ }^{6}$. We are developing imaging methods to rapidly obtain high-resolution 3D x-ray CT volumes of excised rat lungs and analysis methods to extract quantitative measurements from the image data which reflect both the structure and mechanical properties of the vessels in the pulmonary arterial tree.

*correspondence: email: Roger.Johnson@Marquette.edu; (414)288-7841; fax: (414)288-7938

http://semblance.bien.mu.edu/ molthen/Imaging//magingGroup.html 


\section{MATERIALS AND METHODS}

We built a micro-CT scanner consisting of an $\mathrm{x}$-ray tube, a specimen stage and an imaging detector ${ }^{13,1}$. The tube is a demountable microfocal unit providing high-resolution projections when a magnification geometry is employed. The small specimen is placed in close proximity to the source with a large format detector situated relatively far away ${ }^{15,16}$. The precision stage translates and rotates the specimen in the conebeam of $x$ rays. The detector is a high-resolution $9-7-5^{\prime \prime}$ image intensifier coupled with a tandem optical system to a $1024^{2}$-pixel, low noise CCD camera. For rat lung imaging, we typically operate the source at $85 \mathrm{kVp}$ and 30 microamps tabe current, with minimal $(0.5-\mathrm{mm}$ beryllium) inherent filtration. Currently it takes three minutes to acquire 360 views at $512^{2}, 8$-bit resolution during a full rotation of the tat lung.

We image contrast-enhanced lungs from control rats and experimental rats in which pulmonary hypertension has been induced by exposure to chronic hypoxia ( $11 \% \mathrm{O}_{2}$ for three weeks). The rats are anesthetized, the trachea and pulmonary artery cannulated and the lungs excised. The vessels are flushed with physiological saline solution until cleared of blood, then the lungs suspended by the camulas from the top of a thin-walled polyacetate cylinder as can be seen in Figure 1 where the cannulas are just visible at the

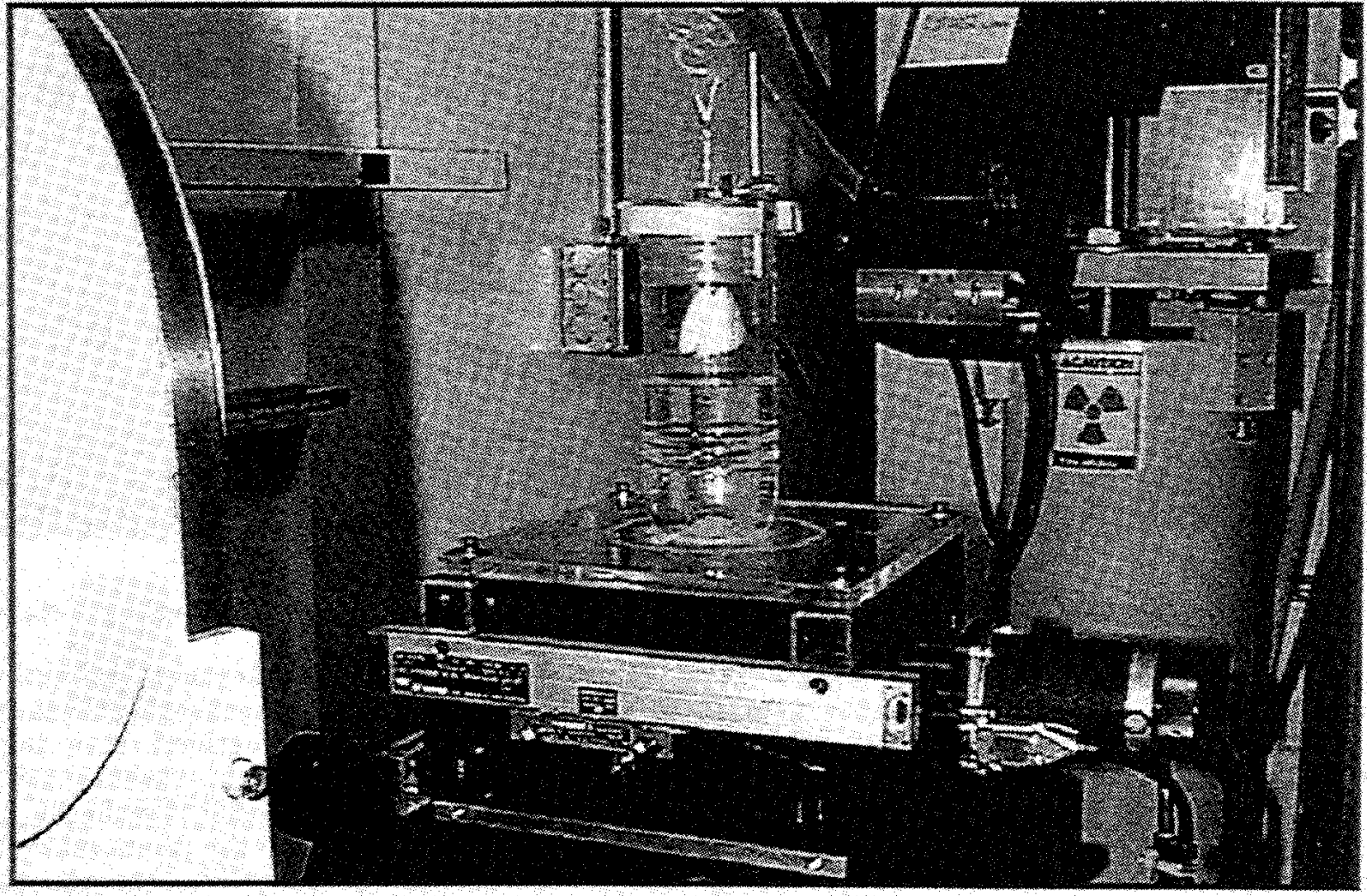

Figure 1: Photo of micro-CT scanner with rat lung in place for imaging. $X$-ray source is to the right, specimen in the middle with arterial and tracheal cannulas visible, and image intensifier to the left.

top. A brominated perfluorocarbon (Perflubron; perfluorooctyl bromide) contrast agent is then introduced through the arterial cannula. Due to the surface tension at the perfluorocarbon-aqueous interface, when introduced into the pulmonary artery, the contrast agent fills only the arterial side and does not enter the capillaries or veins. Thus, we can maintain the contrast agent at a variety of static intra-arterial pressures spanning the physiological range. We image either whole lungs or tied-off left lung lobes at magnifications ranging from $3.5 \mathrm{X}$ to $9 \mathrm{X}$. 
Projection preprocessing includes correcting the pincushion distortion of the image intensifier using an unwarping technique in which the polynomial coefficients are determined from the image of a precision grid of ball bearings ( $\mathrm{BB}$ phantom $)^{17.99}$. The rotation axis is centered using a sinogram, and the illumination nonuniformity corrected by floodfield division. Images are reconstructed onto a $512^{3}$ grid using the Feldkamp conebeam filtered backprojection algorithm ${ }^{20}$ with a Shepp-Logan filter ${ }^{21}$. Since the contrast agent is much denser than the bulk of the lung, the reconstructed data can be fairly reliably segmented, for example to facilitate creation of surface shaded displays, using a seeded region growing segmentation algorithm. The user specifies the minimum size of connectivity list to be accepted in the segmentation (the smallest connected region falling within a specified gray scale window which is to be accepted as vessel). Thus, the binary volume becomes a segmented volume of the connected vascular tree. and almost all spurious background noise is eliminated from the binary result. Figure 2 shows an asacquired projection of a rat lung in the middle panel. The corresponding surface-shaded rendering from the same view angle is shown on the right with a rendering from another representative angle to the left. Our renderings are produced using IDL (Interactive Data Language) software ${ }^{22}$. The rat lung structure would fit within a $2.5 \mathrm{~cm}$ sphere, and was imaged at a magnification of about $4 X$ using the $7^{\prime \prime}$ image intensifier input.

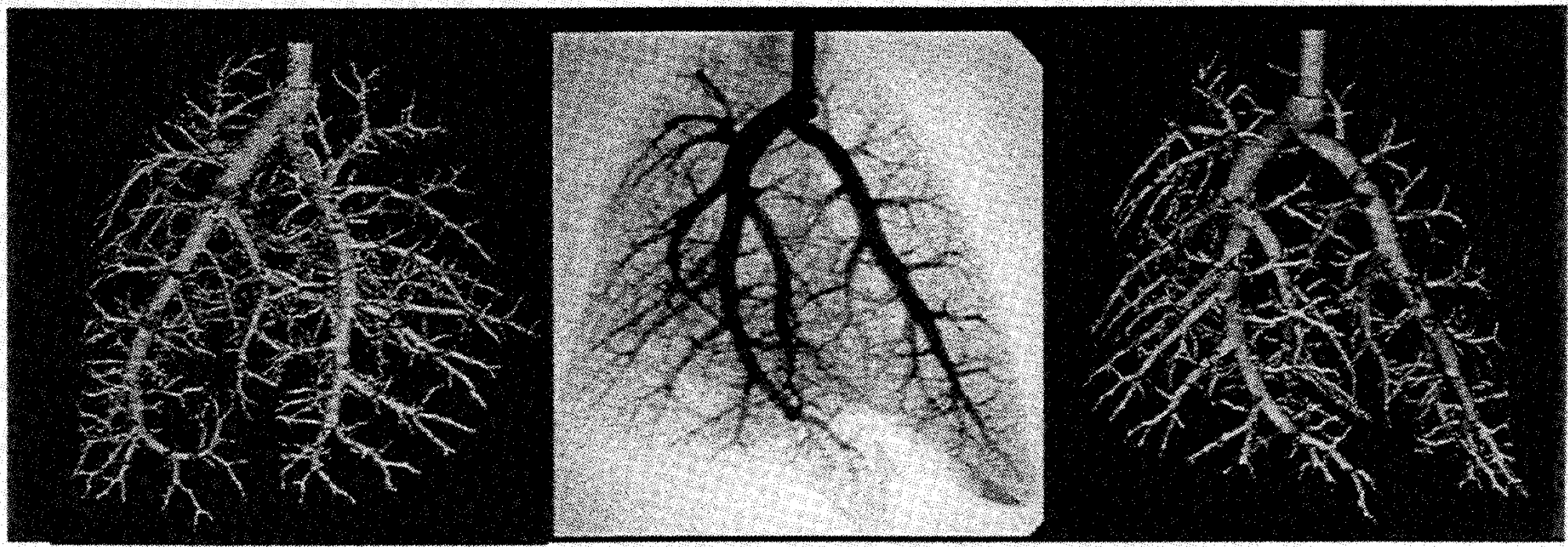

Figure 2: Left: Surface-shaded rendering of reconstruction of rat pulmonary arterial tree.

Middle panel: As-acquired rat lung projection. Right: Rendering of the reconstruction viewed from the same angle.

One of the problems with quantitative analysis of a structure as complex at the pulmonary arterial tree is the large volume of data available in the images, prompting us to explore rational procedures for data reduction. In the past several methods have been used to summarize the morphometric data derived from arterial casts, including centrifugal and centripetal ordering schemes. In centrifugal ordering, vessels are classified according to generation proceeding from the pulmonary artery to the periphery ${ }^{23}$ while in centripetal ordering (for example using the Strahler method) the classification begins with the precapillary arterioles, and proceeds up the tree with the order increasing toward the pulmonary artery ${ }^{24.25}$. Despite the various ordering systems used, published data ${ }^{26-30}$ from several species appear to be quite consistent with respect to linearity and slope if one plots, for example, the log of the number of segments having a certain diameter vs. log segment diameter or the log of segment length vs. log segment diameter. This property of pulmonary arterial tree geometry suggests altematives to the problem of morphometric summary.

Ritman developed method, based on analysis of main trunks of the arterial tree, which is particularly useful in the context of volumetric $\mathrm{CT}$ data ${ }^{31}$. Their results suggest that a reasonable number of measurements of segment lengths and diameters can be exploited to characterize tree structure. One possible approach is to analyze the longest pathway, or main trunk of the tree from the inlet to the smallest resolvable peripheral vessels. Since this principal pathway contains vessel segments spanning the entire range of diameters 
present in the structure, its geometry might be representative of the tree as a whole. Our results indicate that the various pathways through the tree are self similar in the sense that all subtrees distal to vessel segments of a given diameter are statistically equivalent ${ }^{2}$ and we exploit the self similarity of the tree to extrapolate morphometric and mechanical parameters derived from measurements on a small number of pathways to the structure as a whole.

Accurate measurement of vessel segment diameters is a simple concept, but is complicated in practice by a number of factors related to the physics of the imaging system and nuances of the image analysis and data extraction procedures. Volume averaging causes the densities of vessels less than about wice the width of the imaging system point spread function (PSF), which is degraded by the finite focal spot size, specimen stage imperfections, detector pixel size and the reconstruction algorithm, to be smeared out over a considerably larger volume than they in fact occupy ${ }^{33}$. When we reconstruct transaxial images of subresolution tungsten wires to characterize the system PSF, the cross-sectional image of the wire affects the densities of several pixels (the number of pixels depends upon magnification which is dictated by imaging geometry, even though each pixel represents more than 10 microns, even at the highest magnifications we use.

In the interests of obtaining accurate diameter measurements for small vessels, we developed a model of the ideal circular vessel cross section convolved with the imaging system PSF to calibrate the scanner. Figure 3 illustrates how an ideal vessel lumen cross section larger than twice the full width at half maximum (FWHM) of the system PSF, when convolved with the PSF, yields a density profile whose width at FWHM is a good approximation to the lumen diameter and whose height represents the attenuation characteristics of the contrast agent. A small ideal vessel with width about equal to the PSF, on the other hand, when convolved with the PSF, yields a density trace whose width at FWHM significantly overestimates the vessel diameter and whose height underestimates the contrast agent density. But the areas under the density profiles are proportional to the vessel diameters since, in spite of volume averaging, contrast agent density is conserved in the reconstruction. We have imaged wires and contrast agent-filled tubes of known diameters and found an almost perfectly linear relationship with zero intercept between the area under density profiles taken orthogonal to the wire or tube axis and the true diameter as shown in Figure 4. Thus, as long as at least one cylindrical object of known diameter is contained within the reconstruction, we can accurately measure vessels down to subpixel diameters using a density profile area vs. diameter calibration curve.

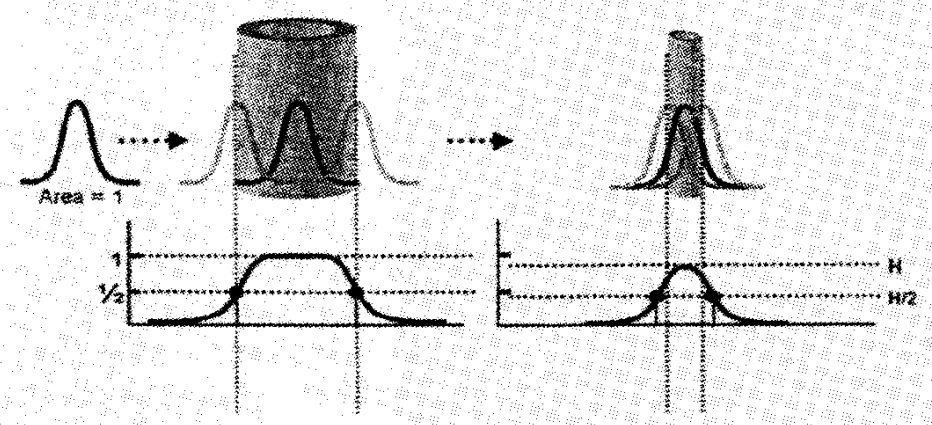

Figure 3: FWHM accurately estimates large vessel diameter but overestimates the small vessel size. But the areas under the density profiles are proportional to the vessel diameters.

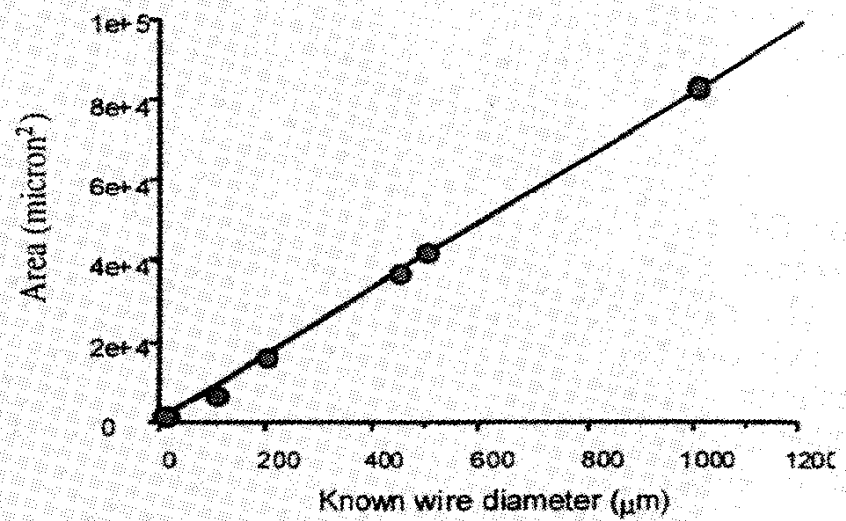

Figure 4: Linear relationship between area under linescan through wire cross section and true wire diameter.

Before developing a reliable calibration method for small vessel diameter measurements, we measured vessels larger than the system PSF using the FWHM criterion applied to the reconstructed image data. In order to measure a statistically significant number of smaller vessels, high magnification planar images of distal portions of the lung were obtained as shown in Figure 5 . Density traces across vessels like the one 
indicated by arrows on the left panel were analyzed, as shown in the right panel, by fitting the linescan data to a model projection of an ideal circular vessel riding on a nonuniform (non-zero) baseline ${ }^{34}$. The width of the model vessel was then taken as the vessel diameter.

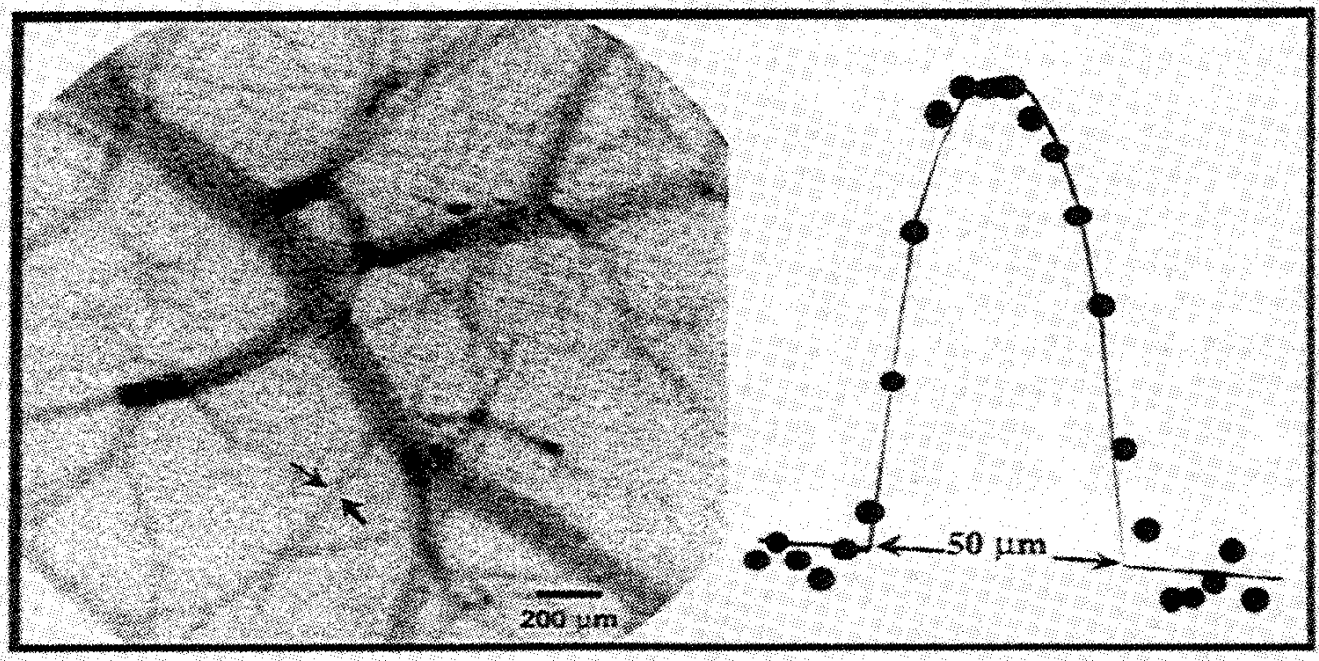

Figure 5: Shows method for measuring small vessel diameters from high-magnification projection inages. Model vessel diameter is indicated as 50 microns in the right panel.

Since our images are reconstructed on cubic-voxel grids of isotropic resolution, it is most straight forward to access axial, sagittal or coronal slices from which to extract density profiles and diameter measurements, as shown for axial slices in the left panel of Figure 6. By interactively "flying through" the stack of transaxial slices, bifurcation locations can be reliably and reproducibly identified as the xyz coordinates
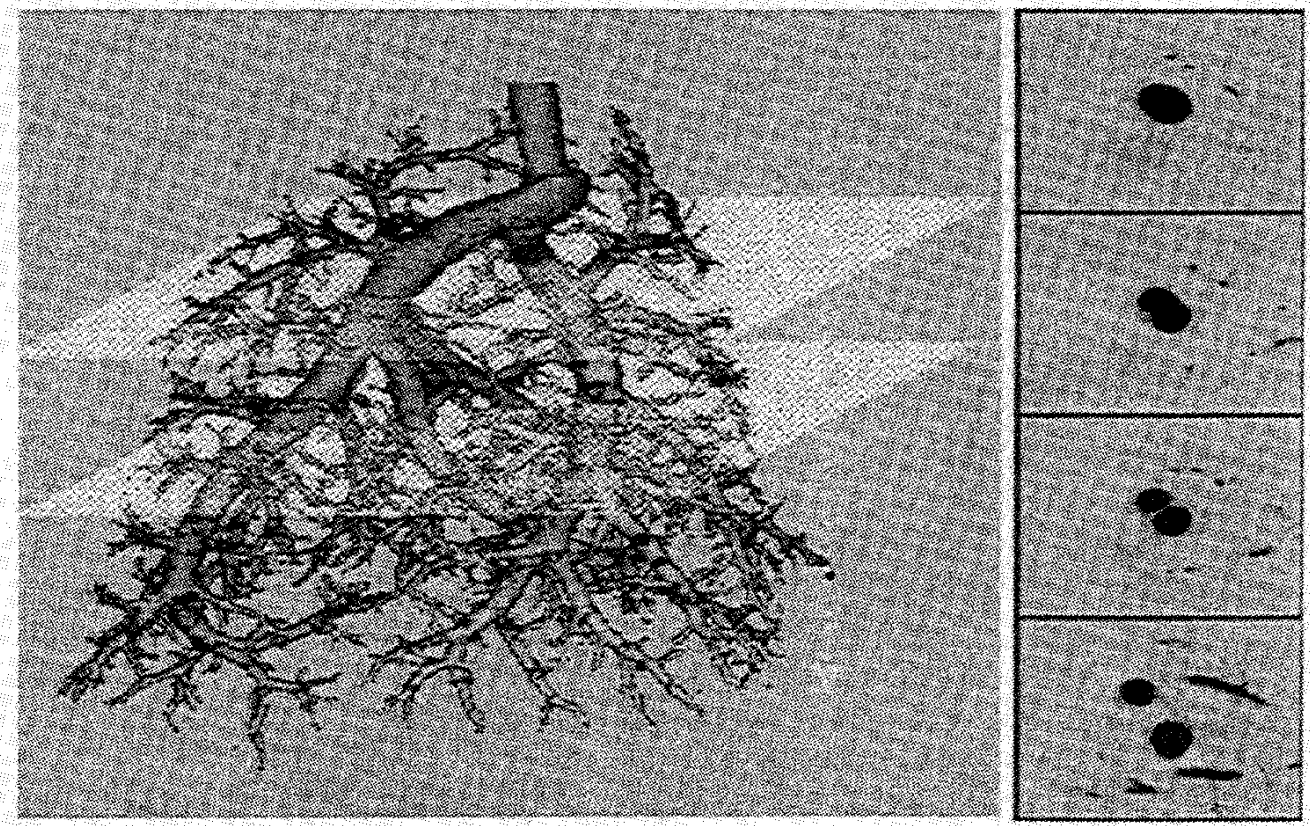

Figure 6: Left: Transaxial slices are readily extracted from the reconstruction.

Right: Bifurcation locations are identified as the voxels where two daughters have just separated as shown in the third panel from top.

where two daughters have just separated as shown on the right of Figure 6 in the third panel from top. After recording the coordinates of all branch points for daughters leaving a pathway, the diameters of the vessel segments comprising the pathway can be measured by accessing the slice equidistant between contiguous branch points defining a segment. However, as is evident in Figure 6 and shown in Figure 7. 
many vessel segment orientations deviate significantly from the vertical. Transaxial and orthogonal slices through a nearly-horizontal vessel are indicated in Figure $7 \mathrm{a}$. The vessel cross section in the transaxial slice appears as a high-aspect-ratio ellipse, as shown at the top in Figure $7 \mathrm{~b}$. It is difficult to make accurate and precise diameter measurements from transaxial image data all the time, so we developed software to access oblique slices orthogonal to vessel segments as illustrated in Figure $7 \mathrm{a}$. The vessel cross section viewed in the orthogonal slice, shown in the bottom panel, Fig. $7 \mathrm{~b}$, is nearly circular, facilitating accurate diameter measurement. Instead of choosing a single radial linescan (density trace) across the orthogonal vessel cross section, the gray-level density surface of the lumen cross section is fit with a circularly-symmetric model function consisting of Gaussians of adjustable standard deviation on each side separated by a rect function, all three of which are variable in height. The heights and the standard deviation of the Gaussian are iteratively adjusted to provide the best least squares fit to the data as shown in Figure $7 \mathrm{c}$. The calibration graph consists of true diameter plotted vs. the areas of cross sections through the fitted Gaussian domes.

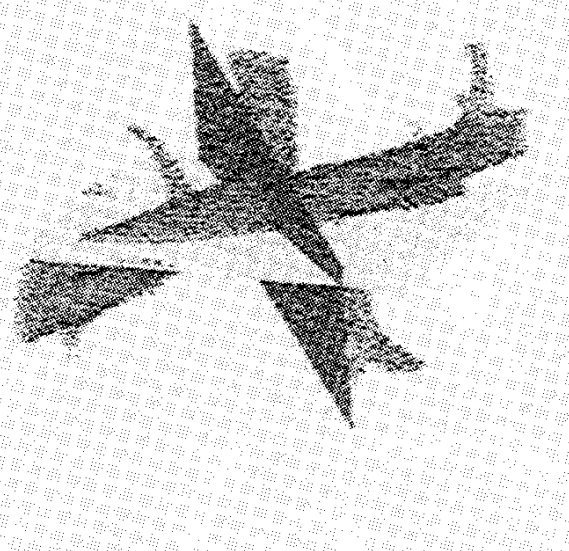

a

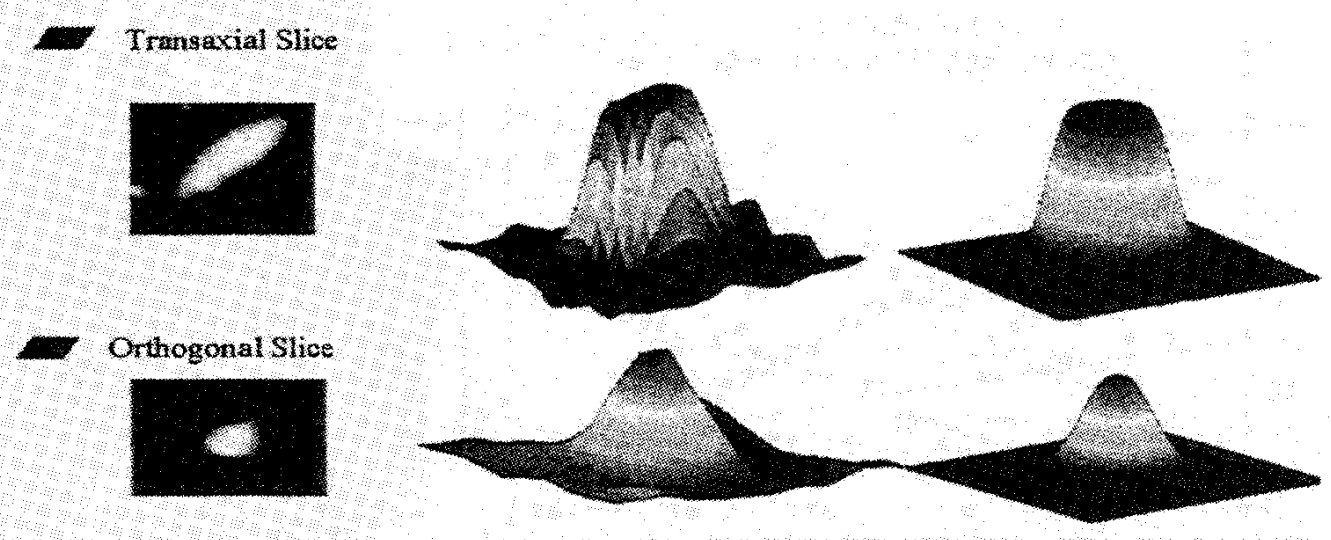

b

$\mathrm{c}$

Figure 7: a) Transaxial (horizontal) and orthogonal slices through a nearly-horizontal vessel are indicated on the left panel. Vessel cross section in the transaxial slice is a high-aspect-ratio-ellipse, as shown in b) top middle panel, whereas the cross section in the orthogonal slice is nearly circular $(b$, bottom). c) Gray-level density surfaces (left) of lumen cross

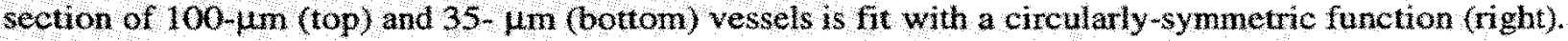

\section{RESULTS}

Our current approach for extracting functionally relevant morphometric parameters from $3 \mathrm{D}$ images of arterial tree structures is to analyze the principal pathway from the inlet to the periphery, measuring the diameters of all segments of the main trunk and of all the daughter branches immediately off the main trunk. The upper curve in Figure 8 a shows a plot of vessel segment diameter (D) vs. distance ( $x$ ) from the pulmonary arterial inlet for the principal pathway of a hypertensive rat lung imaged at high pressure. The lower plot shows the same relationship for the smaller daughter vessel segments branching immediately off the main trunk. Morphometric parameters available from such plots include the parameters of the fitted curves (indicators of slope and curvature) and the dispersion of the data about the fits. The variance is greater for the daughters off the main trunk because the principal pathway tapers fairly steadily from inlet to periphery, while small subtrees may branch off near the large-diameter inlet to perfuse proximal lung tissue volumes. As an example, the data of Figure 9 can be fit to an equation of the form:

$$
D(x)=a(1-b x)^{c}
$$

where $a=D(0)$ is the $y$-intercept, and $b$ and $c$ are parameters of the fit with hemodynamic significance 
since:

$$
\begin{gathered}
b \propto \frac{\left(1-2^{\beta_{2} / \beta_{1}}\right)}{D(0)^{\beta_{2}}} \\
c=\frac{1}{\beta_{2}}
\end{gathered}
$$

and the meaning of $\beta_{1}$ and $\beta_{2}$ is illustrated in Figure $8 \mathrm{~b}$. This figure is a cartoon of three principal pathways, all with the same $\beta_{1}$, which represents the overall taper of the main trunk from inlet to periphery, but each with a different $\beta_{2}$, which determines whether the taper is linear, convex out or concave in. Clearly, these three pathways would exhibit very different hemodynamic characteristics.

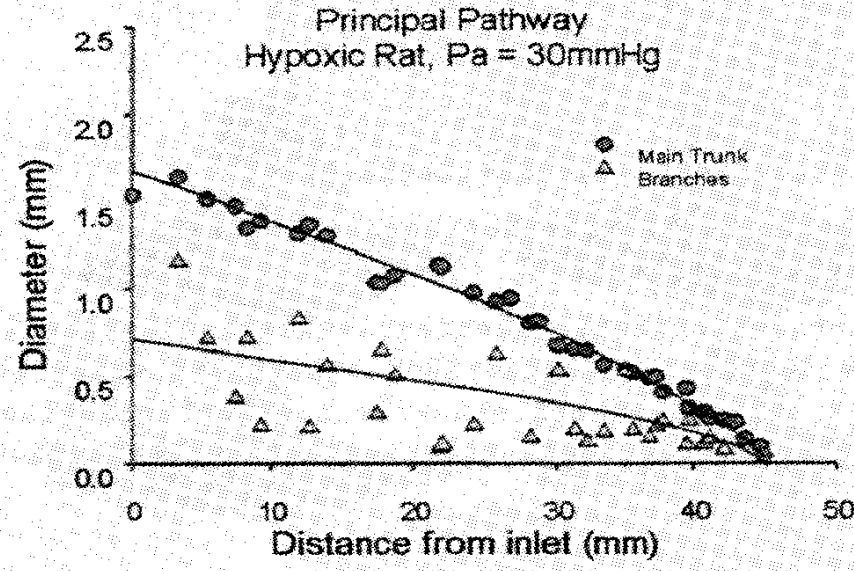

a

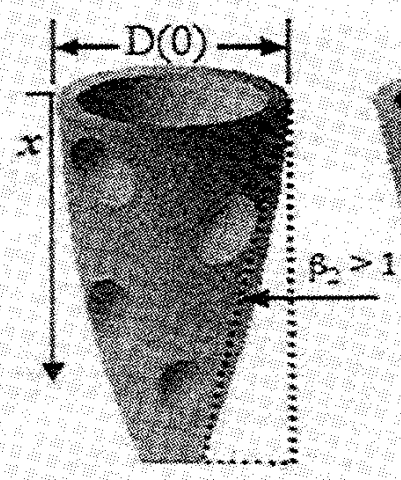

Figure 8: a) Diameter vs. distance plots for main trunk (upper curve) and immediate daughters branching off it (lower curve).

b) Three principal pathways, all with the same $\beta_{1}$ but each with a different $\beta_{2}$.

To assess mechanical properties of the vessels, we obtain $3 \mathrm{D}$ reconstructions of each lung at a series of four intravascular pressures spanning the physiological range $(30,21,12$ and $4 \mathrm{~mm} \mathbf{H g})$. Thus, for every vessel segment measured, the diameter vs. pressure relationship yields the vessel distensibility as percent diameter change per unit change in pressure (\%/Torr). Figure 9 a shows projection images of a hypertensive rat lung obtained at four intra-arterial contrast agent pressures. The distension of the arteries at high pressure is clearly evident.

Figure $9 \mathrm{~b}$ shows plots similar to those in Figure $8 \mathrm{a}$ for the principal pathway of a normal lung imaged at low and high pressures. The slopes, intercepts and curvatures are clearly increased for the control compared to the hypertensive lung, and for the control lung at high compared to low pressure. From the diameter vs, pressure relationships, we can calculate the distensibility (absolute or percent diameter change per torr) of the pulmonary arteries, either for individual vessel segments or for the tree as a whole. The results for a large number of individual vessel segments from several control and several hypertensive lungs are shown in Figure 10a where distensibility is plotted for each vessel segment vs. its diameter at the lowest pressure ( $4 \mathrm{~mm} \mathrm{Hg}$ ). Though the data do overlap, there is reasonably clear separation between the hypertensive and control arterial segments. To calculate the distensibility of the arterial tree as a whole the diameter $(D)$ vs. distance $(x)$ data for all four pressures $(P)$ can be plotted as a three-dimensional graph as shown in Figure $10 \mathrm{~b}$. The data is then fit with a surface of the form:

$$
D(x, P)=a[1+\alpha P(x)](1-b x)
$$


where $\alpha$ is the global distensibility and $a, b$, and $c$ are parameters of the fit. In the case of a normal and a hypertensive rat preliminary results were:

$\begin{array}{lcccc} & \alpha\left(\mathrm{mmHg}^{-1}\right) & \mathrm{a}_{1}(\mathrm{~mm}) & b\left(\mathrm{~mm}^{-1}\right) & \mathrm{c} \\ \text { Normoxic }\left(21 \% \mathrm{O}_{2}\right) & 0.031 & 1.34 & 0.031 & 0.49 \\ \text { Hypoxic }\left(11 \% \mathrm{O}_{2}\right) & 0.021 & 1.26 & 0.033 & 0.44\end{array}$

This shows a significant decrease in the arterial distensibility of the arteries in the diseased lung but no significant change in the other three parameters.

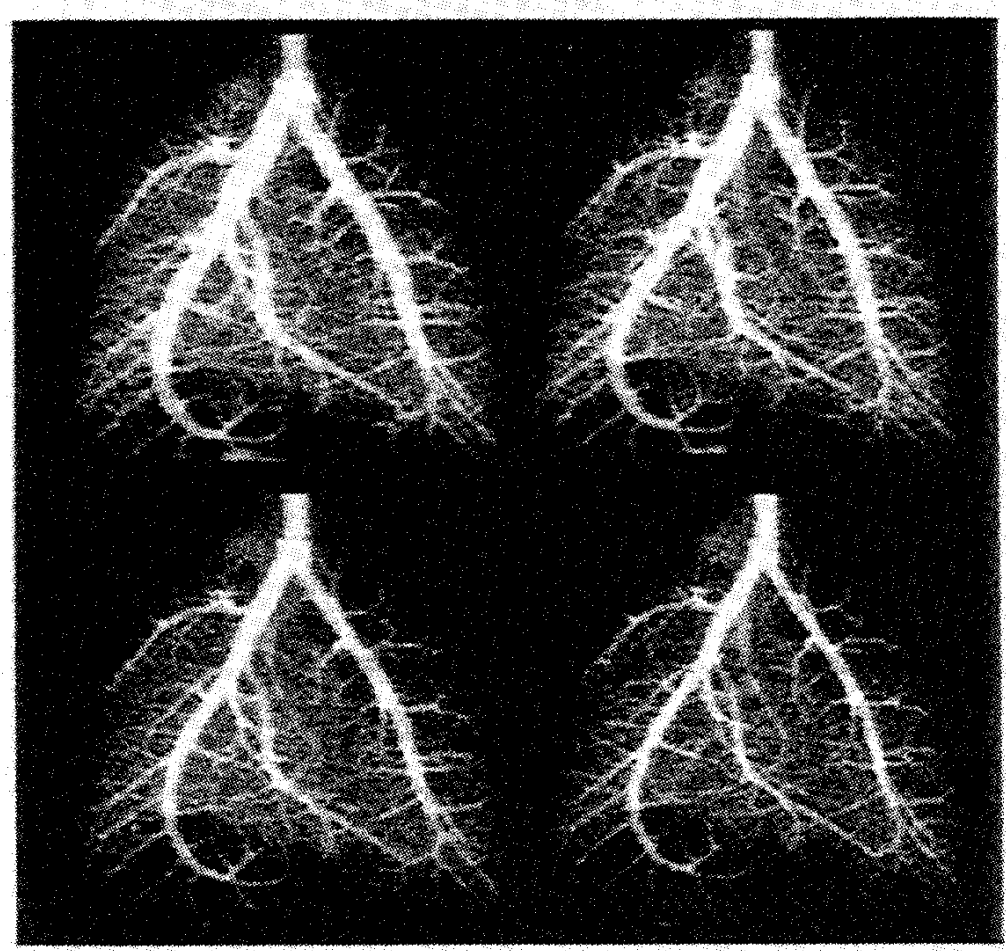

a

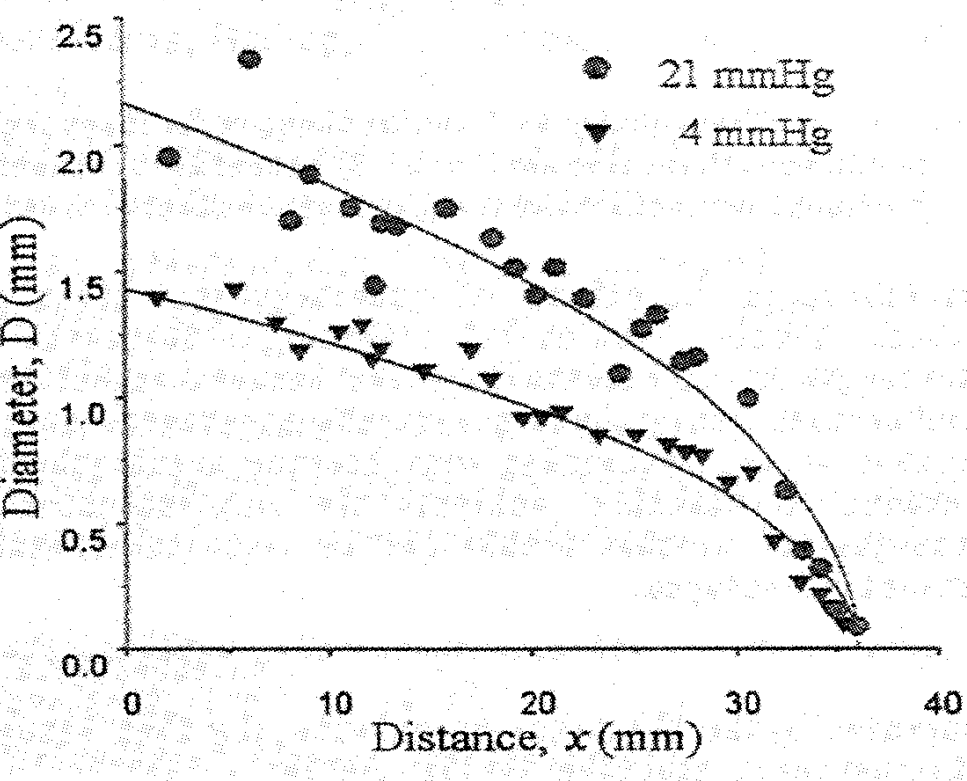

b

Figure 9: a) Projections of rat lung imaged at four intra-arterial contrast agent pressures. b) Diameter vs. distance for principal pathway of a normal rat lung imaged at high and at low pressures.

\section{DISCUSSION}

Micro-CT imaging based methods show promise for rapidly providing quantitative, functionally-relevant measurements of arterial structural and biomechanical properties. The data can be obtained from organs maintained in a physiological state. In the future it may become feasible to perform similar experiments on anesthetized animals, opening up the possibility of longitudinal studies of disease.

Our early results demonstrate that arterial wall distensibility is decreased in hypertensive compared to normal pulmonary trees. Future studies will apply these methods to study the mechanisms involved in other models (such as monocrotaline administration) of pulmonary hypertension, and to studies of the relative efficacies of interventions (such as prostacyclin ${ }^{35}$, calcium channel blockers ${ }^{36}$ or nitric oxide inhalation ${ }^{37}$ ) designed to alleviate symptoms and preferably slow or even reverse pathological remodeling.

Our motivations for these studies include a desire to provide the means for studying changes or differences in pulmonary arterial tree phenotypes in small animal models of pulmonary hypertension so that genetic 


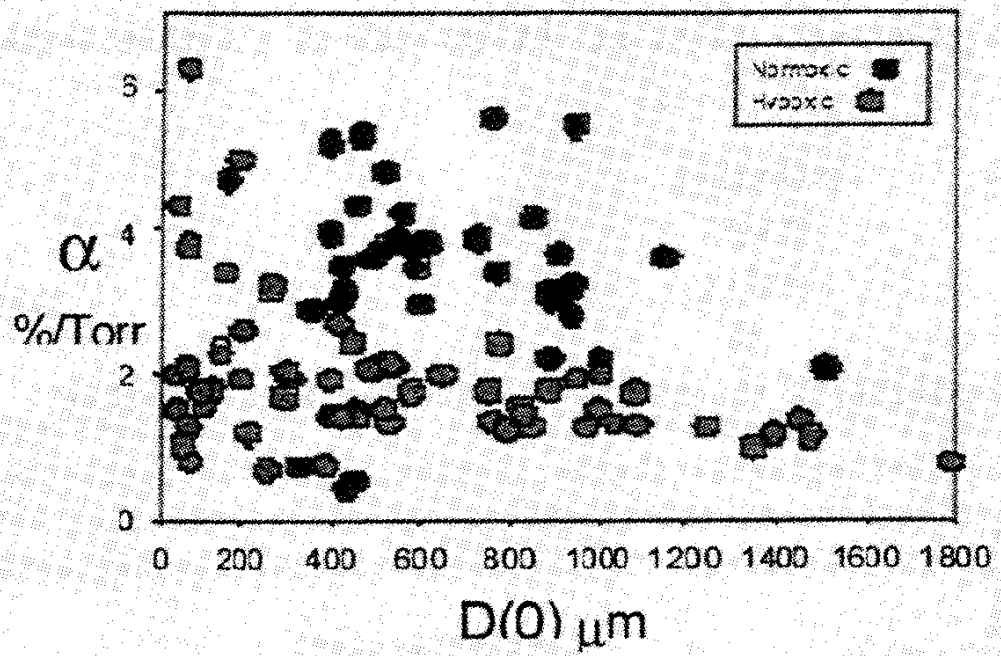

a

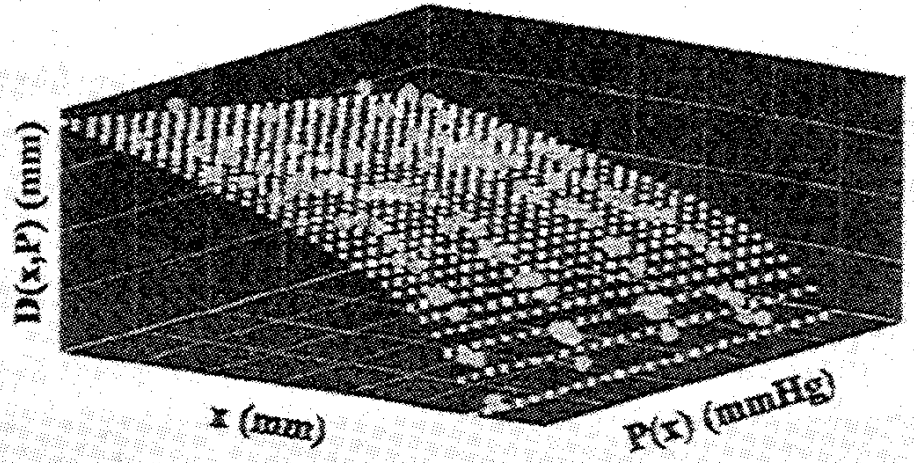

b

Figure 10: a) Distensibility vs. baseline diameter for many individual vessel segments in control and hypertensive pulmonary arterial tree structures. b) Diameter vs. distance data from rat long imaged at four intra-arterial contrast agent pressures. Surface fit yields a global distensibility for the entire arterial tree.

correlations or the effects of conditions that affect putative mechanisms involved in the disease can be studied. A recent twist in the continuing evolution of diagnostic imaging methods has been an emphasis or initiative to develop technology and image analysis methods suitable for high throughput phenotyping of small animals, particularly genetically engineered mice ${ }^{38}$. If they can be made still less labor intensive, the methods we are developing may become applicable to such studies. In addition to providing parameters amenable to statistical analysis, the morphometric parameters could potentially serve as inputs to hemodynamic models which can be used to evaluate the functional significance of remodeling or of aberrant phenotypes.

\section{ACKNOWLEDGEMENTS}

Supported by the Whitaker Foundation, the Falk Medical Trust, the W.M. Keck Foundation, the Veterans Administration, and NHI B H HL-19298.

\section{REFERENCES}

1) Emery, CI, Bee, D, and Barer, GR, "Mechanical Properties and Reactivity of Vessels in Isolated Perfused Lungs of Chronically Hypoxic Rats", Clinical Science 61:569-80, 1981.

2) Finlay, $M$, Barer, $G R$, and Suggett, $A J$, "Quantitative Changes in the Rat Pulmonary Vasculature in Chronic Hypoxia-Relation to Haemodynamic Changes", Quart. J. Exp. Physiol. 71:151-63, 1986.

3) Bergel, DH, "The Static Elastic Properties of the Arterial Wall", $I$ Physiol 156:445-57, 1961.

4) Ivy, DD, Neish, SR, Knudson, OA, Nihill, MR, Schaffer, MS, Tyson, RW, Abman, SH, Shaffer, EM, and Valdes-Cruz, L, "Intravascular Ultrasonic Characteristics and Vasoreactivity of the Pulmonary Vasculature in Children with Pulmonary Hypertension", Am. J. Cardiol 81:740-8, 1998.

5) Caro, CG and Saffman, PG, "Extensibility of Blood Vessels in Isolated Rabbit Lungs", J. Physiol. (London) 178:193-210, 1965 . 
6) Drangova, M, Holdsworth, DW, Boyd, CJ, Dunmore, PJ, Roach, MR, and Fenster, A, "Elasticity and Geometry Measurements of Vascular Specimens Using a High-resolution Laboratory CT Scanner", Physiol. Meas. 14(3):277-90, 1993.

7) Engelberg, $\mathrm{J}$ and BuBois, $\mathrm{AB}$, "Mechanics of Pulmonary Circulation in Isolated Rabbit Lungs", Am. $J$. Physiol. 196:401, 1959.

8) Al-Tinawi, A, Madden, JA, Dawson, CA, Linehan, JH, Harder, DR, and Rickaby, DA, "Distensibility of Small Arteries of the Dog Lung", J. Appl. Physiol. 71(5):1714-22, 1991.

9) Rabinovitch, M, Gamble, W, Nadas, AS, Miettinen, OS, and Reid, L, "Rat Pulmonary Circulation After Chronic Hypoxia: Hemodynamic and Structural Features", Am. J. Physiol. 236 (Heart Circ. Physiol. 5):H818-H827, 1979.

10) Jones, $R$ and Reid, $L$, "Vascular Remodelling in Clinical and Experimental Pulmonary Hypertensions", In: Pulmonary Vascular Remodelling, Bishop, JE, Reeves, JT and Laurent, GJ (eds.), Portland Press, London, 1995, pp.47-115.

11) Dawson, CA, Krenz, GS, Karau, KL, Haworth, ST, Hanger, C, and Linehan, JH, "StructureFunction Relationships in the Pulmonary Arterial Tree", J. Appl. Physiol. 86(2):569-83, 1999.

12) Madden, JA, Al-Tinawi, A, Birks, E, Keller, PA, and Dawson, CA, "Intrinsic Tone and Distensibility of in Vitro and in Situ Cat Pulmonary Arteries", Lung 174:291-301, 1996.

13) Johnson, RH, Wilson, CR, Haworth, ST, Dawson, CA, Linehan, JH, and Roerig, DL, "Design, Modeling and Performance of a Volumetric Micro-CT Scanner", Med. Phys. 24(6):1048, 1997.

14) Johnson, RH, Hu, H, Haworth, ST, Cho, PS, Dawson, CA, and Linehan, JH, "Feldkamp and Circleand-line Conebeam Reconstruction for 3D Micro-CT of Vascular Networks", Phys. Med. Biol. 43(4):929-40, 1998.

15) Nixon, WC, "Point Projection X-ray Microscopy", In: X-ray Microscopy and Microradiography, Cosslett, VE et al. (eds.), Academic Press, New York, 1957, pp.34-45.

16) Cosslett, VE and Nixon, WC, "X-ray Shadow Microscope", Nature 168:24-5, 1951.

17) Cho, PS and Johnson, RH, "Automated Detection of BB Clusters in Digital Fluoroscopic Images", Phys. Med. Biol. 43(9):2677-83, 1998.

18) Wang, $X$ and Ning, $R$, "Accurate and Efficient Image Intensifier Distortion Correction Algorithm for Volume Tomographic Angiography", Opt. Eng. 37(3):977-83, 1998.

19) Fahrig, R, Moreau, M, and Holdsworth, DW, "Three-dimensional Computed Tomographic Reconstruction Using a C-arm Mounted XRII: Correction of Image Intensifier Distortion", Med. Phys. 24(7):1097-1106, 1997.

20) Feldkamp, LA, Davis, LC, and Kress, JW, "Practical Cone-beam Algorithm", J. Opt. Soc. Am. 1(6):612-9, 1984.

21) Shepp, LA and Logan, BF, "The Fourier Reconstruction of a Head Section", IEEE Trans. Nuc. Sci. 21:21-43, 1974.

22) Research Systems, Boulder, Colorado.

23) Weibel, ER, "Lung Morphometry and Models in Respiratory Physiology", In: Respiratory Physiology An Analytical Approach, Chang, HK and Paiva, M (eds.), Marcel Dekker, New York, 1989, pp.1-56. 
24) Horsfield, K, "Functional Morphology of the Pulmonary Vasculature", In: Respiratory Physiology An Analytical Approach, Chang, HK and Paiva, M (eds.), Marcel Dekker, New York, 1989, pp.499-531.

25) Singhal, $S$, Henderson, $R$, Horsfield, $K$, Harding, $K$, and Cumming, G, "Morphometry of the Human Pulmonary Arterial Tree", Circ. Res. 33:190-7, 1973.

26) Jiang, ZL, Kassab, GS, and Fung, YC, "Diameter-defined Strahler System and Connectivity Matrix of the Pulmonary Arterial Tree", J. Appl. Physiol. 76:882-92, 1994.

27) Yen, RT, Zhuang, FY, Fung, YC, Ho, HH, Tremer, H, and Sobin, SS, "Morphometry of the Cat's Pulmonary Arterial Tree", J. Biomech. Eng. 106:131-6, 1984.

28) Miller, WS, "The Structure of the Lung", J. Morphol. 8:165-88, 1893.

29) Gan, RZ and Yen, RT, "Vascular Impedance Analysis in Dog Lung with Detailed Morphometric and Elasticity Data", J. Appl. Physiol. 77:706-17, 1994.

30) Horsfield, K, "Morphometry of the Small Pulmonary Arteries in Man", Circ. Res. 42(5):593-7, 1978.

31) Liu, Y-H and Ritman, EL, "Branching Pattern of Pulmonary Arterial Tree in Anesthetized Dogs", $J$. Biomech. Eng. 108:289-93, 1986.

32) Johnson, RH, Karau, KL, Molthen, RC, and Dawson, CA, "Exploiting Self-similarity of Arterial Trees to Reduce the Complexity of Image Analysis", SPIE Vol. 3660, Physiology and Function from Multidimensional Images, Clough, AV and Chen, C-T (eds.), San Diego, February 20-26, 1999, pp.351-361.

33) Dougherty, G and Newman, D, "Measurement of Thickness and Density of Thin Structures by Computed Tomography: A Simulation Study", Med. Phys. 26(7):1341-8, 1999.

34) Clough, AV, Krenz, GS, Owens, M, Al-Tinawi, A, Dawson, CA, and Linehan, $\mathrm{JH}$, "An Algorithm for Angiographic Estimation of Blood Vessel Diameter", J. Appl. Physiol. 71(5):2050-8, 1991.

35) Higenbottam, T, "Pathophysiology of Pulmonary Hypertension A Role for Endothelial Dysfunction", Chest 105(2):7S-12S, 1994.

36) McLaughlin, VV and Rich, S, "Medical and Surgical Approaches to Pulmonary Hypertension", Comp. Ther. 23(9):612-6, 1997.

37) Shirai, M, Shimouchi, A, Kawaguchi, AT, Sunagawa, K, and Ninomiya, I, "Inhaled Nitric Oxide: Diameter Response Patterns in Feline Small Pulmonary Arteries and Veins", Am. J. Physiol. 270(Heart Circ. Physiol. 39):H974-80, 1996.

38) Lin, MC, Rockman, HA, and Chien, KR, "Heart and Lung Disease in Engineered Mice", Nature Medicine 1(8):749-51, 1995. 